\title{
Variational Monte Carlo studies of a $t-J$ model on an anisotropic triangular lattice
}

\author{
Tsutomu Watanabe ${ }^{1,2 *}$, Hisatoshi Yokoyama ${ }^{3}$, Yukio TanakA ${ }^{1,2}$, Jun-ichiro Inoue $^{1}$ and Masao Ogata ${ }^{4}$ \\ ${ }^{1}$ Department of Applied Physics, Nagoya University, Nagoya 464-8603 \\ ${ }^{2}$ CREST Japan Science and Technology Corporation (JST) \\ ${ }^{3}$ Department of Physics, Tohoku University, Sendai 980-8578 \\ ${ }^{4}$ Department of Physics, University of Tokyo, Bunkyo-ku, Tokyo 113-0033
}

\begin{abstract}
With the insulating phase of $\kappa$-(BEDT-TTF $)_{2} \mathrm{X}$ in mind, we study a $t$ - $J$ model on an anisotropic triangular lattice, where the hopping integral is $t^{\prime}$ in one of the three directions, using a variational Monte Carlo method. By changing the value of $t^{\prime} / t$, we study the stability of superconducting (SC) states with $d$ - and $d+i d$-wave symmetries and of an antiferromagnetic (AF) state. As $t^{\prime} / t$ decreases from 1 , the stable state immediately switches from the $d+i d$ wave to the $d$ wave. The AF state is stabilized from the normal spin liquid state for $t^{\prime} / t \lesssim 0.7$ at half filling. We also take account of Nagaoka ferromagnetism and a phase separation.
\end{abstract}

KEYWORDS: $t$ - $J$ model; anisotropic triangular lattice; variational Monte Carlo method; $d+i d$ wave

\section{Introduction}

Recent discoveries of superconductivity in $\kappa$-(ET) $)_{2} \mathrm{X}$ (ET: BEDT-TTF $)^{1}$ and $\mathrm{Na}_{x} \mathrm{CoO}_{2} \cdot y \mathrm{H}_{2} \mathrm{O}^{2}$ are of high interest for some reasons. They are strongly correlated electron systems $(2 p-\pi$ or $3 d$ orbitals) with quasi-twodimensional lattice structures, like high- $T_{\mathrm{c}}$ cuprates. However, they differ from the cuprates in that the lattice structure is essentially triangular, and thus the effect of frustration can play a leading role for superconductivity as well as magnetism. For such lattices, we expect novel superconducting (SC) features, distinguished from the cuprates on the square lattice. In particular, identification of pairing symmetry is a primary subject.

Recently, we studied the stability of SC states for the $t-J$ model on the isotropic triangular lattice, using a variational Monte Carlo (VMC) method, ${ }^{3}$ which accurately treats the local constraint of no double occupation. According to it, irrespective of the sign of $t$, a $d_{x^{2}-y^{2}}+i d_{x y}$ wave is the most stable among various pairing symmetries near half filling, in accordance with mean-field-type approximations. ${ }^{4}$ At half filling, the simple $d$ wave is degenerate with the $d+i d$ wave. In contrast, weak-coupling approaches which consider more realistic band structure of $\mathrm{Na}_{x} \mathrm{CoO}_{2} \cdot y \mathrm{H}_{2} \mathrm{O}$ - a reduced single band ${ }^{5}$ or multi bands ${ }^{6}$ - led to different symmetries.

In contrast, $\kappa-(\mathrm{ET})_{2} \mathrm{X}$ seems to be better described using single-band models on an anisotropic triangular lattice, where the hopping integral in two of the three directions is $t$ and that in the remaining direction $t^{\prime}$. According to the band calculations for $\kappa$-ET salts, ${ }^{7,8}$ the value of $t^{\prime} / t$ is often near unity with $t>0$. So far, theoretical studies using the fluctuation exchange (FLEX) approximation for the Hubbard model ${ }^{9}$ have concluded that the pairing symmetry is a simple $d_{x^{2}-y^{2}}$ wave like the cuprates. The results of $\mathrm{NMR}^{10}$ showed that the superconductivity is unconventional with nodes, but the pairing symmetry is not necessarily $d$ wave.

In this work, we extend our preceding study ${ }^{3}$ to an anisotropic triangular lattice or a $t-t^{\prime}-J-J^{\prime}$ model, which connects the square lattice $\left(t^{\prime} / t=0\right)$ and the isotropic triangular lattice $\left(t^{\prime} / t=1\right)$. The main purpose of this paper is to compare the stability between the $d$ - and $d+i d$ wave pairing states, when the values of $t^{\prime} / t$ and carrier density $\delta(1-n)$ are changed. At half filling $(\delta=0)$, the trial wave functions become insulating for the $t-J$ model, but the gap parameter $\Delta_{k}$ for the SC state remains finite; thus the favorable form of $\Delta_{k}$ can be determined for this singlet liquid (or RVB) state. Actually, $\kappa$-ET salts, whose electron density is often at half filling, bring about a superconductor-insulator transition, when their effective value of $U / t$ ( $U$ : onsite repulsion) is increased through (chemical) pressure. ${ }^{1}$ Since such a transition is properly described using a Hubbard-type model, as shown for the square lattice, ${ }^{11}$ we will leave it for a coming publication.

In addition, we also consider the possibility of Nagaoka ferromagnetism (FM) and a phase separation. For the isotropic case with $t<0$, the Nagaoka FM defeats the SC states in a wide range of $n$ and $J /|t|(0 \leq \delta \lesssim 0.96$ and $0 \leq J / t \lesssim 0.6) .^{3,12}$ It is still controversial whether the phase separation takes place or not for very small $J / t$ and $\delta \sim 0$ for the square lattice. We thus trace the development of these two states with changing $t^{\prime} / t$.

\section{Formulation}

According to the comparison of experimental results with band calculations for the $\kappa$-(ET) $)_{2} \mathrm{X},{ }^{7,8}$ the ET molecules in the conducting planes are strongly dimerized. If each dimer makes a single lattice site, the conducting planes can be properly described with a $t-J$ model on an anisotropic triangular lattice (or an extended square lattice with next-nearest-neighbor pairs in only one diagonal direction $[1,1]$ ) as follows,

$$
\begin{aligned}
H= & -\sum_{\langle i, j\rangle \sigma} t_{i j} P_{\mathrm{G}}\left(c_{i \sigma}^{\dagger} c_{j \sigma}+\text { H.c. }\right) P_{\mathrm{G}} \\
& +\sum_{\langle i, j\rangle} J_{i j}\left(\mathbf{S}_{i} \cdot \mathbf{S}_{j}-\frac{1}{4} n_{i} n_{j}\right),
\end{aligned}
$$




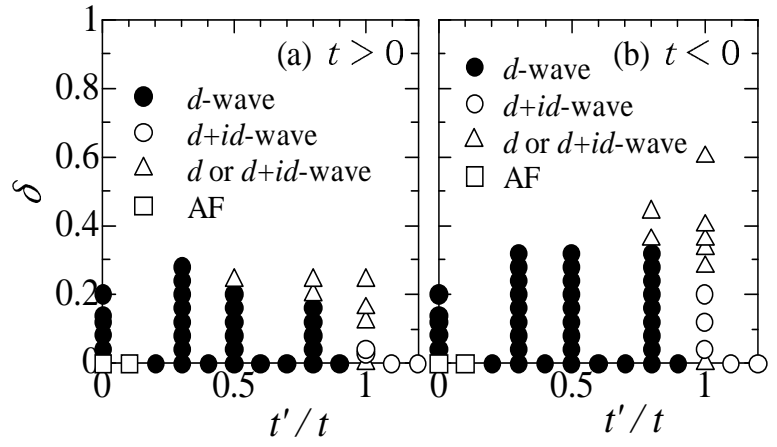

Fig. 1. The most stable state among $d$ - and $d+i d$-wave and AF states is shown in the $t^{\prime} / t-\delta$ space. Here, $J /|t|=0.3$, and the systems are $10 \times 10$ and $12 \times 12$. (a) $t>0$ and (b) $t<0$.

where $P_{\mathrm{G}}=\prod_{i}\left(1-n_{i \uparrow} n_{i \downarrow}\right)$. Here, $t_{i j}=t$ and $J_{i j}=J$ for the nearest-neighbor-site pairs in two of the three lattice directions, and $t_{i j}=t^{\prime}$ and $J_{i j}=J^{\prime}$ for the nearest-neighbor-site pairs in the remaining direction, and $t_{i j}=J_{i j}=0$ otherwise. In view of the relation with the Hubbard model, $J=4 t^{2} / U$, we put $J^{\prime} / J=\left(t^{\prime} / t\right)^{2}$. For $\kappa$-(ET $)_{2} \mathrm{X}$, the value of $t^{\prime} / t$ is estimated as $0.5-1.1$, and the electron density is mostly at half filling. In this paper, we consentrate on the case of $n \leq 1$, because a case of $n>1$ can be mapped to a case of $n<1$ with the different signs of $t$ and $t^{\prime}$ through a particle-hole transformation.

For this model, we perform VMC calculations, ${ }^{13}$ which precisely treat the local constraint the $t$ - $J$ model imposes. As a variational wave function, the Gutzwiller type $\left(\Psi=P_{G} \Phi\right)$ becomes a good starting point for $t-J$ type models. ${ }^{14}$ We use $\Psi_{\mathrm{n}}=P_{\mathrm{G}} \Phi_{\mathrm{F}}$ for the normal state $\left(\Phi_{\mathrm{F}}\right.$ : Fermi sea $), \Psi_{\mathrm{SC}}=P_{\mathrm{G}} \Phi_{\mathrm{BCS}}$ for the superconducting state $\left(\Phi_{\mathrm{BCS}}\right.$ : a BCS function with a $\mathbf{k}$-dependent gap $\left.\Delta_{\mathbf{k}}=\Delta z_{\mathbf{k}}\right)$, and $\Psi_{\mathrm{AF}}=P_{\mathrm{G}} \Phi_{\mathrm{AF}}$ for the $\operatorname{AF}$ state $\left(\Phi_{\mathrm{AF}}:\right.$ a Hartree-Fock state with an AF gap $\Delta$ ). In this paper, we focus on the $d$ wave $\left(z_{\mathbf{k}}=\cos k_{x}-\cos k_{y}\right)$ and the $d+i d$ wave $\left(z_{\mathbf{k}}=\cos k_{x}+e^{i 2 \pi / 3} \cos \left(k_{x}+k_{y}\right)+e^{i 4 \pi / 3} \cos k_{y}\right)$, because other symmetries are unstable in the regime of our concern for both the isotropic case ${ }^{3}$ and the square lattice. ${ }^{15}$ In $\Psi_{\mathrm{SC}}$ and $\Psi_{\mathrm{AF}}, \Delta$ is a sole variational parameter, which becomes nonzero when $\Psi$ has a long-range order, except for the case of $\Psi_{\mathrm{SC}}$ at half filling $(\delta=0)$.

In this study, we collect samples as many as $10^{5}$ $10^{7}$, which reduce the error in energy to approximately $10^{-4}|t|$. We use the systems of $N_{\mathrm{s}}=8 \times 8-12 \times 12$ with periodic-antiperiodic boundary conditions and electron densities satisfying the closed shell condition.

\section{Results}

Let us start with the phase diagram in the $t^{\prime} / t-\delta$ plain shown in Fig. 1, which is constructed by comparing the energies among the $d$ - and $d+i d$-wave and AF states for $J /|t|=0.3$. As studied in, ${ }^{3}$ for the isotropic case $\left(t^{\prime} / t=\right.$ $1)$, the $d+i d$ wave is the most stable near half filling, but the $d$ wave has an indistinguishably close energy for $\delta=0$ and low densities. When $t^{\prime} / t$ is reduced from 1 , the stable symmetry rapidly switches to the $d$ wave (solid circles), which prevails in a wide range of $\delta$ and $t^{\prime} / t$ including the

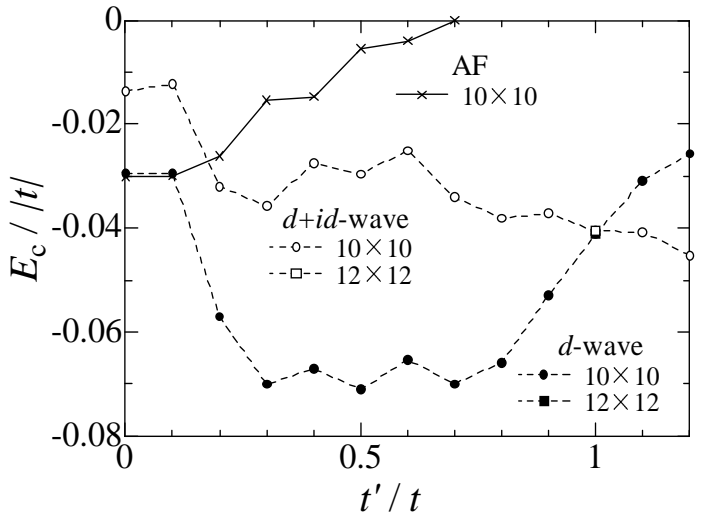

Fig. 2. Energy reduction by the $d$ - and $d+i d$-wave spin liquid (or RVB) states and the AF state as a function of $t^{\prime} / t$ at half filling $(\delta=0)$. The systems are $10 \times 10$ and $12 \times 12$. Note that nonsmooth behavior of $E_{\mathrm{c}}$ chiefly stems from the discrete $\mathbf{k}$-points of finite systems; when the value of $t^{\prime} / t$ changes, occupied $\mathbf{k}$-points (or Fermi surface) discontinuously changes for the normal and AF states.

square lattice, regardless of the sign of $t$.

To consider the half-filled case more in detail, we plot the energy difference, $E_{\mathrm{c}}=E_{\mathrm{SC}}-E_{\text {normal }}$, as a function of $t^{\prime} / t$ in Fig. 2. Since both $\Psi_{\mathrm{n}}$ and $\Psi_{\mathrm{SC}}$ are insulating for $\delta=0, E_{\mathrm{c}}(<0)$ indicates the energy reduction by forming a singlet liquid (or RVB) state. ${ }^{16}$ When $t^{\prime} / t$ decreases, $E_{\mathrm{c}}$ for the $d$ wave considerably decreases, whereas $E_{\mathrm{c}}$ for the $d+i d$ wave tends to increase. Thus, the $d$ wave becomes widely stable, especially for $t^{\prime} / t \sim 0.5$. On the other hand, for $t^{\prime} / t>1$, the $d+i d$ wave becomes more stable, inversely. The result of dominant $d$-wave symmetry is basically the same with those of the previous FLEX studies $^{9}$ for SC states.

For the square lattice, the AF state $\Psi_{\mathrm{AF}}$ is slightly more stable than the $d$-wave RVB state $\Psi_{\text {SC }}$ at half filling, and becomes unstable as soon as holes are doped. ${ }^{15}$ Even if $t^{\prime} / t$ is introduced as in eq. (1), the cases in which the AF state is the most stable are limited to half filling with $t^{\prime} / t=0$ and 0.1 , as shown in Fig. 1. This is partly due to the fact that the $t$ - $J$ model tends to underrate the stability of the AF state, because the AF state becomes stable in the weaker-coupling region than the $d$ wave does. ${ }^{11}$ However, energy reduction by $\Psi_{\mathrm{AF}}$ survives up to $t^{\prime} / t=0.7$ (see Fig. 2). Considering, in addition, that for $t^{\prime} / t=0$ and $0 \leq \delta \lesssim 0.1$, a coexisting state of $\mathrm{AF}$ and $d$-wave RVB orders is stable, ${ }^{17}$ the $d$-wave RVB state at half filling may simultaneously exhibit an $\mathrm{AF}$ order in some range of $t^{\prime} / t(>0)$. For $t^{\prime} / t>0.7$, it is probable that a Mott insulator with another spin order, e.g. a $120^{\circ}$-structure Néel order, ${ }^{18}$ appears. We leave these issues for future studies.

Next, we discuss Nagaoka FM, ${ }^{19}$ which is a complete FM appearing in general for $J /|t| \sim 0$ and $\delta \sim 0$. For the square lattice, its region is fairly narrow, namely, $0<$ $\delta \lesssim 0.4$ and $J / t \lesssim 0.1 .^{20}$ In the isotropic case, the region of FM vanishes for $t>0$, but considerably expands to $0 \leq \delta \lesssim 0.96$ and $J /|t| \lesssim 0.7$ for $t<0 .{ }^{3,12}$ In Figs. 3, we depict phase diagrams for three values of $t^{\prime} / t$. The 

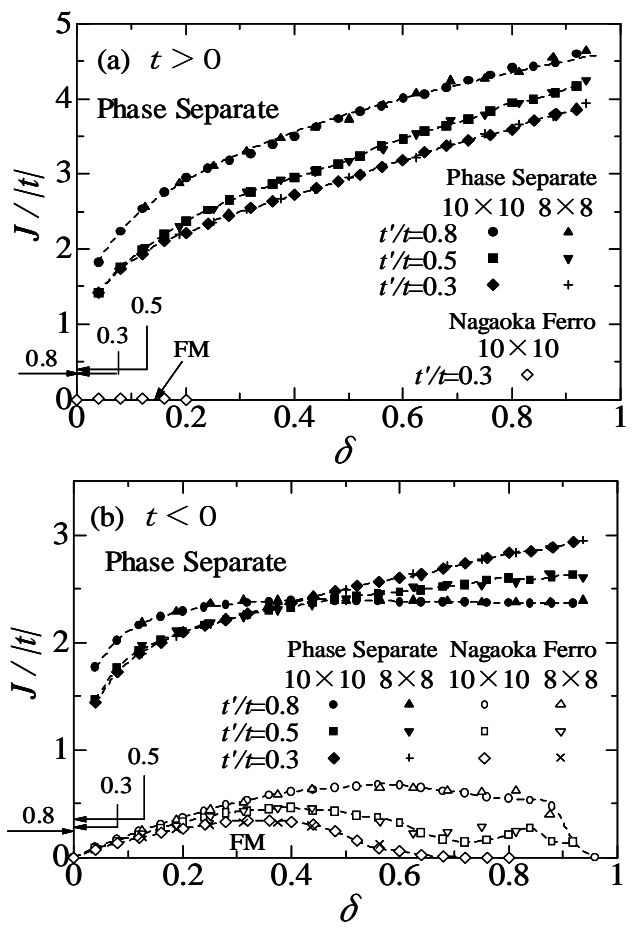

Fig. 3. Phase diagram in the $\delta-J /|t|$ space for the phase separation and the complete FM for (a) $t>0$ and (b) $t<0$. For $t^{\prime} / t=0.3$, the range of complete FM with $t>0$ is $0 \leq \delta \lesssim 0.2$ and $J /|t| \lesssim 0.015$. The arrows on the vertical axis indicate the critical values of the phase separation estimated by the way (ii).

boundaries are determined by comparing the energies of complete FM (spinless fermion) and the $d$ wave (VMC), which is the most stable SC state. For $t>0$, as $t^{\prime} / t$ increases, the range of FM rapidly reduces, and vanishes for $t^{\prime} / t=0.5$, whereas for $t<0$, the range of FM rapidly expands at first, and becomes almost the same area as the isotropic case for $t^{\prime} / t=0.8$. Thus, FM does not seem to appear for $t>0$, whereas for $t<0$ the Nagaoka FM is likely to expel the SC state from the realistic parameter region even for small $t^{\prime} / t$.

Finally, we consider a phase separation. Due to the exchange term, the $t$ - $J$-type models necessarily bring about a phase separation for large values of $J /|t|$. Here, we estimate the boundary between the $d$-wave and phase separation in two ways. (i) We suppose that the inhomogeneous phase separates into the two hole densities, $\delta=0$ (half filling) and $\delta=1$ (empty), and the energy of this state is approximated as $E^{\mathrm{PS}}=(1-\delta) E^{d}$, where the $E^{d}$ is optimized total energy of the $d$ wave at half filling. The boundary is determined by comparing $E^{\mathrm{PS}}$ and $E^{d}(\delta)$. Although we use $E^{d}$ instead of $E^{\text {exact }}$ because the latter is not known for general values of $t^{\prime} / t$, the results are connected without a marked discrepancy to the cases $t^{\prime} / t=0^{15}$ and $1,^{3}$ where $E^{\text {exact }}$ is available. (ii) We estimate the boundary near half filling, using the condition of intrinsic stability, $\partial^{2} E / \partial \delta^{2}>0$, for the $d$ wave. Near half filling, this approximation is better.

The results of (i) and (ii) are summarized in Figure 3. For $t>0$, the critical value $J_{\mathrm{c}} /|t|$ linearly increases as $\delta$ increases for large $\delta$, and the slope is slightly suppressed, as $t^{\prime} / t$ decreases. For $t<0$, as $t^{\prime} / t$ decreases, the slope of $J_{\mathrm{c}} /|t|$ becomes steeper. Regardless of the signs of $t$ and the value of $t^{\prime} / t$, the estimated value of $J_{\mathrm{c}} /|t|$ near half filling is small enough to be realistic. Thus, the possibility of phase separation remains near half filling, but this is not the case for intermediate and large values of $\delta$.

\section{Conclusion}

We have studied a single-band $t$ - $J$ model on an anisotropic triangular lattice, based on variational Monte Carlo calculations. It is revealed that (1) within the superconducting states, the plain $d_{x^{2}-y^{2}}$ wave is dominant in almost the entire range of $t^{\prime} / t$ and $\delta$ near half filling. (2) At half filling, the $d$-wave RVB state is more stable than the $d+i d$ wave for $t^{\prime} / t<1$, while the relation is opposite for $t^{\prime} / t>1$; the AF state is stabilized from the normal spin liquid for $t^{\prime} / t \lesssim 0.7$. (3) For $t<0$, even when the value of $t^{\prime} / t$ is small, the Nagaoka ferromagnetism occupies a wide range of $\delta$ and $J /|t|$. (4) Regardless of the values of $t^{\prime} / t$, the phase separation may arise near half filling, but not for intermediate and large values of $\delta$.

In this work, we have considered a $t$ - $J$-type model for the insulating phase of $\kappa-(\mathrm{ET})_{2} \mathrm{X}$. However, we need to tackle a Hubbard-type model to study a superconductorinsulator transition as well as a superconducting phase itself at half filling, like ${ }^{11}$ for the square lattice. At the final stage in preparing this paper, we became aware of a VMC study for a Hubbard model. ${ }^{21}$

\section{Acknowledgments}

This work is partly supported by Grant-in-Aids from the Ministry of Education, Culture, Sports, Science and Technology, by the Supercomputer Center, ISSP, University of Tokyo, NAREGI Nanoscience Project, Ministry of Education, Culture, Sports, Science and Technology, Japan, which enables us to carry out the calculations on the computers at the Research Center for Computational Science, Okazaki National Research Institutes, and a Grant-in-Aid for the 21st Century COE "Frontiers of Computational Science".

1) R.H. Mckenzie, Science 278 (1997) 820; K. Kanoda, Physica C 282-287 (1997) 299.

2) K. Takada, H. Sakurai, E. Takayama-Muromachi, F. Izumi, R.A. Dilanian and T. Sasaki, Nature 422 (2003) 53.

3) T. Watanabe, H. Yokoyama, Y. Tanaka, J. Inoue and M. Ogata: to appear in J. Phys. Soc. Jpn. 73 vol. 12 (2004).

4) G. Baskaran, Phys. Rev. Lett. 91 (2003) 097003; B. Kumar and B.S. Shastry, Phys. Rev. B 68 (2003) 104508; M. Ogata, J. Phys. Soc. Jpn. 72 (2003) 1839.

5) Y. Nishikawa, H. Ikeda and K. Yamada: J. Phys. Soc. Jpn. 73 (2004) 1127; Y. Tanaka, M. Ogata and Y. Yanase, J. Phys. Soc. Jpn. 73 (2004) 319; K. Kuroki, Y. Tanaka and R. Arita: Phys. Rev. Lett. 93 (2004) 077001.

6) W. Koshibae and S. Maekawa: Phys. Rev. Lett. 91 (2003) 257003; Y. Yanase, K. Mochizuki and M. Ogata: condmat/0407563.

7) T. Komatsu, N. Matsukawa, T. Inoue and G. Saito, J. Phys. Soc. Jpn. 65 (1996) 1340.

8) R.H. MacKenzie, Cond. Mat. Phys. 18 (1998) 309.

9) J. Schmalian, Phys. Rev. Lett. 81 (1998) 4232; H. Kino and H. Kontani, J. Phys. Soc. Jpn. 67 (1998) 3691; H. Kondo and T. Moriya, J. Phys. Soc. Jpn. 67 (1998) 3695.

10) H. Mayaffre, P. Wzietec, D. Jerome, C. Lenoir and P. Batail, Phys. Rev. Lett. 75 (1995) 4122; S. M. De Soto, C.P. Slichter, A.M. Kini, H.H. Wang, U. Geiser and J.M. Williams, 
Phys. Rev. B 52 (1995) 10364; K. Kanoda, K. Miyagawa, A. Kawamoto and Y. Nakazawa, Phys. Rev. B 54 (1996) 76.

11) H. Yokoyama, Y. Tanaka, M. Ogata and H. Tsuchiura, J. Phys. Soc. Jpn. 73 (2004) 1119.

12) T. Koretsune and M. Ogata: Phys. Rev. Lett. 89 (2002) 116401, and J. Phys. Soc. Jpn. 72 (2003) 2437.

13) D. Ceperley, G.V. Chester and K.H. Kalos, Phys. Rev. B 16 (1977) 3081; H. Yokoyama and H. Shiba, J. Phys. Soc. Jpn. 56 (1987) 1490.

14) H. Yokoyama and H. Shiba, J. Phys. Soc. Jpn. 59 (1990) 3669; A. Paramekanti, M. Randeria and N. Trivedi, Phys. Rev. Lett. 87 (2001) 217002.

15) H. Yokoyama and M. Ogata: J. Phys. Soc. Jpn. 65 (1996)
3615.

16) P. W. Anderson, P. A. Lee, M. Randeria, T. M. Rice, N. Trivedi and F. C. Zhang, J. Phys. Cond. Mat. 16 (2004) R755.

17) T. Giamarchi and C. Lhuillier, Phys. Rev. B 43 (1991) 12943; A. Himeda and M. Ogata, Phys. Rev. B 60 (1999) R9935.

18) L. Capriotti, A.E. Trumper and S. Sorella, Phys. Rev. Lett. 82 (1999) 3899.

19) Y. Nagaoka: Phys. Rev. 147 (1966) 392.

20) H. Yokoyama and H. Shiba: J. Phys. Soc. Jpn. 56 (1987) 3570; W. O. Putikka, M. U. Luchini and M. Ogata: Phys. Rev. Lett. 69 (1992) 2288.

21) J. Liu, J. Schmalian and N. Trivedi, cond-mat/0411044. 